\title{
Is There Something Quantum-Like about the Human Mental Lexicon?
}

\author{
Peter Bruza \\ Faculty of Science and Technology \\ Queensland University of Technology \\ p.bruza@qut.edu.au
}

\begin{abstract}
This talk proceeds from the premise that IR should engage in a more substantial dialogue with cognitive science. After all, how users decide relevance, or how they chose terms to modify a query are processes rooted in human cognition. Recently, there has been a growing literature applying quantum theory (QT) to model cognitive phenomena. This talk will survey recent research, in particular, modelling interference effects in human decision making. One aspect of QT will be illustrated - how quantum entanglement can be used to model word associations in human memory. The implications of this will be briefly discussed in terms of a new approach for modelling concept combinations. Tentative links to human abductive reasoning will also be drawn. The basic theme behind this talk is QT can potentially provide a new genre of information processing models (including search) more aligned with human cognition.
\end{abstract}

Acknowledgments. This research is supported in part by the Australian Research Council Discovery grant DP0773341. 